\title{
Performance Test for Rapid Surface Modeling of Dynamic Construction Equipment from Laser Scanner Data
}

\author{
C. Wang ${ }^{a}$ and Y.K. Cho ${ }^{b}$ \\ ${ }^{a}$ Ph.D. Candidate, School of Civil and Environmental Engineering, Georgia Institute of Technology, Mason \\ Building 3136, 790 Atlantic Dr., Atlanta, GA 30332-0355 \\ ${ }^{b}$ Associate Professor, School of Civil and Environmental Engineering, Georgia Institute of Technology, Mason \\ Building 4140A, 790 Atlantic Dr., Atlanta, GA 30332-0355 \\ E-mail: cwang2@gatech.edu,yong.cho@ce.gatech.edu
}

\begin{abstract}
-
In modern dynamic construction fields, more attention has been paid on safely operating heavy construction equipment such as cranes, excavators, and concrete pump. In order to improve the safety in complicated jobsites, it is highly required to provide heavy equipment operators with accurate measurement of on-site objects in near real time. In this paper, a rapid surface modeling method and its performance evaluation through on-site tests are introduced. The performance of the proposed method was tested with a heavy equipment at construction site. The interrelationships among data size, processing time, and the size of the resultant hull segments were examined from the data analyses. The field experimental results demonstrate that the proposed dynamic surface modeling method would significantly improve the equipment operation productivity and safety by distinguishing a dynamic surface model being controlled by the operator from the point cloud of existing static environment in 3D views.
\end{abstract}

Keywords -

Construction equipment; Point cloud; Object recognition; 3D modeling; Safety

\section{Introduction}

Visibility-related accidents can be easily caused by the interactions between workers, equipment, and materials. This problem can lead to serious collisions without pro-active warnings. There have been a number of advances in vision-aid techniques because lacking full visibility is a major contributing factor in accidents at construction sites. $3 \mathrm{D}$ spatial modeling can help to optimize equipment control [1, 24], significantly improve safety [2-3], monitor construction progress [4], and enhance a remote operator's spatial perception of the workspace [5-8]. However, the rapid processing of tens of thousand bits of range data in real time is still an unsolved problem requiring further investigation [9]. Unstructured work areas like construction sites are difficult to graphically visualize because they highly involve unpredictable activities and change rapidly. Construction site operations require real-time or near real-time information about the surrounding work environment, which further complicates graphical modeling and updating.

One commonly used method to obtain the $3 \mathrm{D}$ position of an object is based on $3 \mathrm{D}$ laser scanning technology [7, 10-11]; this method, however, has some limitations, such as low data collection speed and low object recognition rates [12]. It has always been a challenge to recognize specific objects from a $3 \mathrm{D}$ point cloud in unstructured construction environments because it is difficult to rapidly extract the target area from background scattered noises in a large and complex 3D point cloud.

While rapid workspace modeling is essential to effectively control construction equipment [13], few approaches have been accepted by the construction industry due to the difficulty of addressing all the challenges of current construction material handling tasks with the current sensor technologies. Thus, an innovation in rapid 3D spatial information is necessary to meet the challenges. The main objective of this paper was to validate a $3 \mathrm{D}$ visualization framework to collect and process dynamic spatial information rapidly at a construction job site for safe and effective construction equipment operations. Multi-video camera integrated vision-based object recognition and tracking method has been developed, based on which, a smart laser scanning method was proposed to reduce data size and scanning time. 


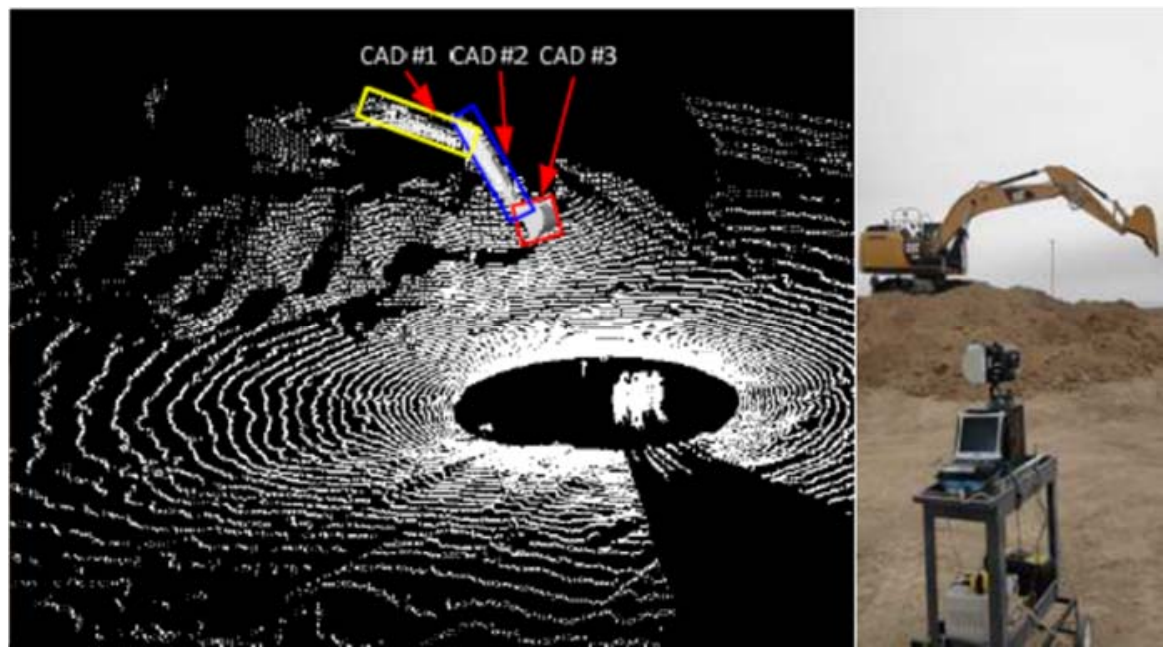

Fig. 1. Model-based object recognition and registration [17]

\section{Related Work}

For the operator to monitor blind spots of the workspace from the cab, a vision-based system using a single or multiple cameras is an inexpensive option [14]. Brilakis et al. [15] introduced 2D vision-based methods that recognize new overlapping feature points and track them in the subsequent video stream. To acquire a precise $3 \mathrm{D}$ position of objects with additional depth information, generally two or more cameras generate a stereo view after calibration with known intrinsic parameters. Park et al. [16] achieved more accurate 3D locations of tracking objects by projecting the centroids of the tracked entities from two cameras to 3D coordinates. Notwithstanding the recent advances, there are some known drawbacks of vision-based techniques in tracking moving equipment at the sites: 1) additional infrastructure is needed to install and maintain cameras; 2) fixed camera locations have limited view angles and resolutions, and 3 ) the results are sensitive to lighting conditions [17].

Laser scanners have been extensively utilized to automatically obtain the "as-is" condition of the existing buildings [18]; they also can be used to classify and capture a complex heavy equipment operation as it happens or to provide automated feedback to those who are conducting the operations [7, 17, 19]. Teizer et al. presented a methodology for real-time 3D modeling using Flash LADAR which has a limited measurement range and low accuracy for outdoor use [3]. Lee et al. proposed an automated lifting-path tracking system on a tower crane to receive and record data from a laser device [13]. Bosche and Hass registered 3D static CAD objects to laser-scanned point cloud data [20], which can be utilized to efficiently assess construction processes. However, most of the algorithms were developed mainly to recognize and register static objects' models to point clouds. Few applications have demonstrated the technical feasibility of registering dynamic models to point clouds in real or near real time. In the authors' previous studies [17], a model-based automatic object recognition and registration method, Project-Recognize-Project (PRP), was introduced to register the $\mathrm{CAD}$ models with the corresponding point cloud of the recognized objects through comparing the recognized point cloud of the objects with existing CAD models in a database (shown in Fig. 1.). While the PRP approach provides very detailed, accurate solid models in a point cloud, the limitation of this method is that it only works for the objects which have corresponding models in the database. In this study, a non-model based, surface modeling method is introduced to automatically recognize and visualize dynamic objects on construction sites.

\section{Overview of the Proposed Method}

In Fig. 2, the framework of the proposed rapid surface modeling method is illustrated. The developed data acquisition system is composed of two $2 \mathrm{D}$ line laser scanners ( 80 meter working ranges at $100 \mathrm{~Hz}$ scan speed, up to $2.5 \mathrm{sec} / 360^{\circ}$ scan, $190^{\circ}$ for vertical line), a digital camera and three video cameras with a resolution of 0.25 degree in a vertical direction and 0.0072 degree in a horizontal direction. In this system, multiple 


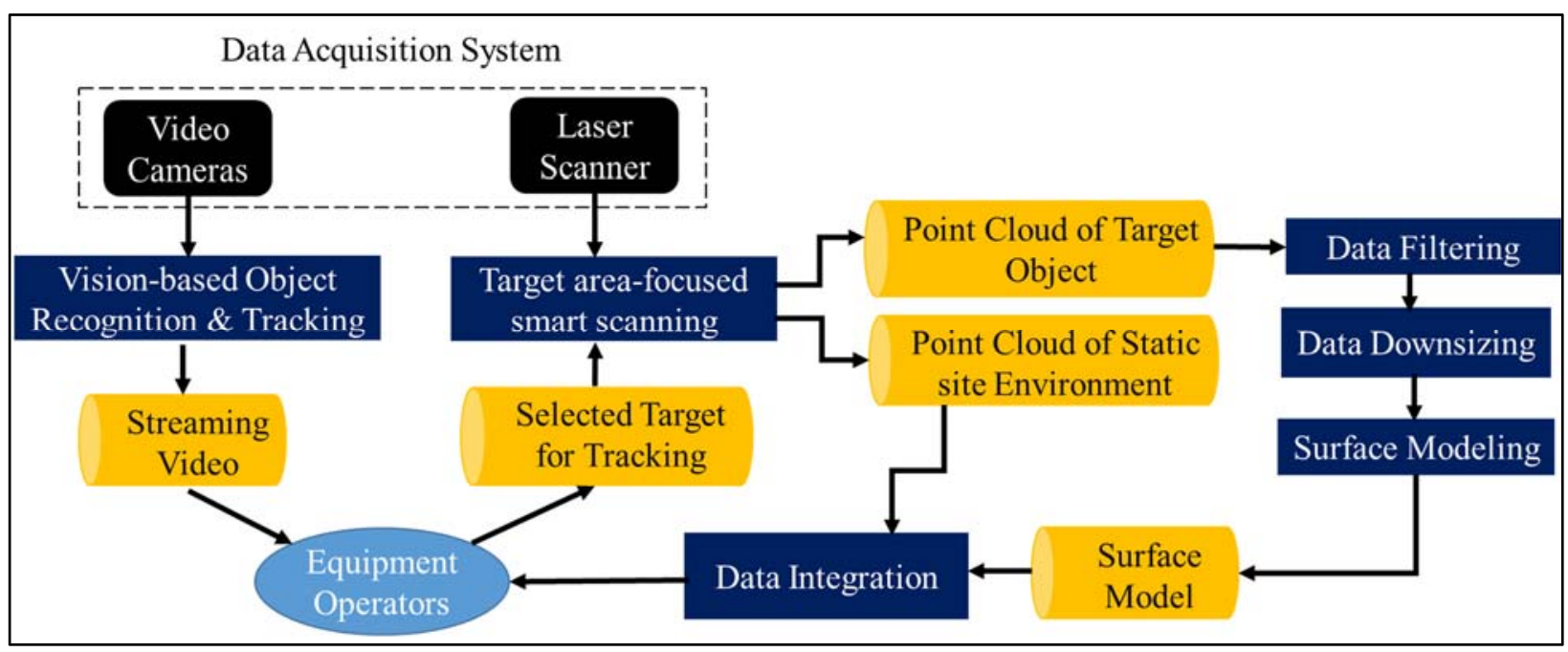

Fig. 2. The framework of the proposed method

degree-of-freedom (DOF) kinematic problems were solved based on the mechanical installation, and 3D point cloud data and digital image streams can be collected simultaneously. Utilizing this form of flexible design together with Time-of-flight (TOF) laser scanner working type, higher scanning resolution and faster scanning rate were obtained, which is more suitable for the complicated dynamic construction environments.

Working together with the laser scanner system, the digital video cameras were employed to capture real time image streams in the jobsite for the operators. Then, the operators can select the target objects in the image streams by drawing a bounding box through the developed graphical user interface. Using a robust local feature detector, Speeded Up Robust Features (SURF) [21], image-based target object recognition and tracking algorithms were implemented.

Taking the bounding box area as an input, smart scanning and visualization processes were immediately applied to separately collect and update the data of the target object and the static site environment. The major two steps of the smart scanning method are: (1) a static jobsite environment is scanned by the laser system with a very high resolution. Those collected site data are stored in the memory; and (2) from the second scanning round, only the point clouds in the dynamic target area specified by the equipment operator are updated separately. In this process, the size of point cloud data obtained from complex large construction sites and the updating time can be significantly reduced.

Surface modeling of point clouds can be more advantageous for the equipment operators over the point-cloud-only visualization because a surface model of target object can be better distinguished from a complex point-cloud environment. The concave hull approach is one of the most widely used surface modeling methods creating a polygon that represents the area occupied by a set of points. A concave hull better details the shape of the point cloud than the convex hull does. A concave hull of a set of surfaces is the enclosing concave surfaces with smallest volume. There are several existing concave hull calculation algorithms [22], however the efficiency of these algorithms decreases significantly because of high computing queries for the large size of point cloud data like the ones obtained from construction jobsites.

The raw data size of point cloud data collected from a construction jobsite is quite a large and as a result, it is challenging to process and visualize these data in real or near real time. In this study, as shown in Fig. 4, the smart scanning technology was employed at the very beginning of the surface modeling phase in order to significantly reduce the data size of the point clouds and the surface modeling time. In addition, outliers of the point data were statistically identified and removed to improve concave hull model accuracy. Then, a data filtering and downsizing process was conducted to further decrease the number of points. The goal of data downsizing is to increase the data processing speed by reducing the amount of overly dense data being processed.

\section{Field Test and Discussion}

Validation of the proposed methodology was implemented on a backhoe loader at a building construction site. The data acquisition system was mounted on a mobile cart, and set up in the working area of the equipment, especially in its blind spots. It firstly scanned the whole jobsite and kept the point cloud data in the database; then smart scanning and visualization were fulfilled based on the selected tracking objects. All tests were benchmarked on an Intel Core i5 CPU with 4GB RAM on a 64 bit Windows 
mobile computer. It should be noted that the required resolution, registration accuracy, and scan rate for successful surface modeling vary based on the scan range, ambient lighting conditions, properties of the target (e.g., shape, color, reflectivity, and moving speed), and the number of mounted $2 \mathrm{D}$ laser scanner. In this study, all data were collected from the system with two 2D laser scanners, and the maximum scan speed of which is 227,500 points/sec. The scan speed could be doubled if four 2D laser scanners were equipped [17].

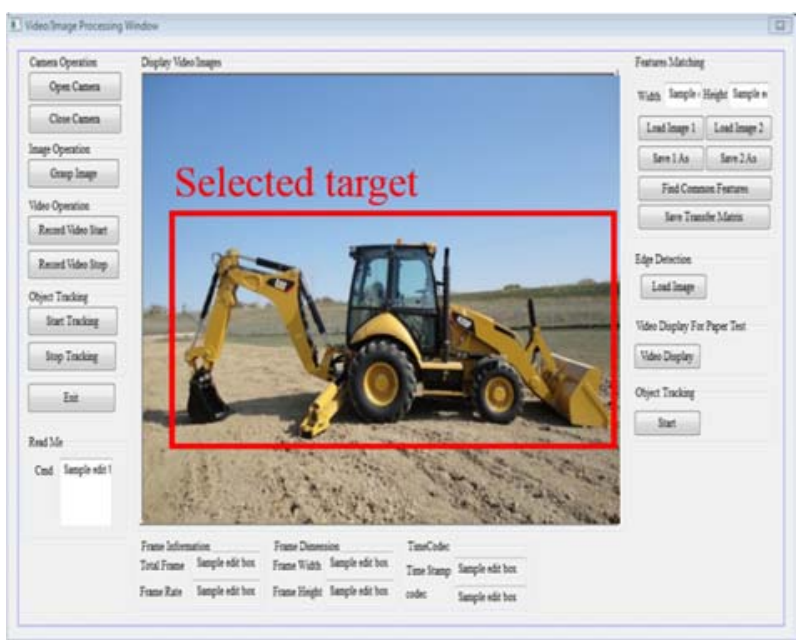

Fig. 3 The backhoe loader was chosen as target in the developed graphical user interface

In this study, a backhoe loader was chosen as a test subject. The entire equipment was selected as the tracking target, as shown in Fig. 3. Through smart scanning, the point cloud of the target area and the site environment were separately collected and visualized in Fig. 4., and 18,304 points were collected from the target. Then the collected raw data was processed by the data filtering and downsizing algorithms to decrease the surface modeling time. The resolution (the average point to point distance) of the raw data is $0.01 \mathrm{~m}$, and the raw data size became 17,585 after filtering, and varied from 61 to 17,311 after downsizing based on different leaf sizes (Fig. 5. and Table 1). The leaf size here is defined as the edge length of the $3 \mathrm{D}$ voxel, and the greater the leaf size is, the more the data size is decreased. Table 2 shows the processing time under different data downsizing scales and the size of the concave hull segments. In Table 2, $\alpha$ limits the size of the resultant concave hull segments. The smaller $\alpha$ is, the more detailed the hull segments are. The concave hull surface modeling on raw data without data filtering and downsizing was also evaluated. As shown in Table 3 , the range of surface modeling time for raw data was between 1 to 4 seconds. Thus, data filtering and downsizing was needed before surface modeling to reduce the data size for a real-time application. In Fig. 6 , the total processing time includes time for data filtering, downsizing, and surface modeling. When the leaf size was $0.01 \mathrm{~m}$, the total processing time was greater than 1 second, but in the other sizes the time was all within 1 second. To deliver a best available result to the operator, therefore, the smallest $\alpha$ value was selected. Fig. 7 shows the surface modeling result of the backhoe holder integrated with the data of the static site environment.

Table 1. Data size of each step of the proposed methodology

\begin{tabular}{|l|l|c|}
\hline \multicolumn{2}{|l|}{} & $\begin{array}{c}\text { Number of } \\
\text { points }\end{array}$ \\
\hline Raw data & 18,304 \\
\hline Filtered data & 17,585 \\
\hline \multirow{2}{*}{} & Leaf size $=\mathbf{5 0} *$ Resolution* & 61 \\
\cline { 2 - 3 } & Leaf size $=\mathbf{1 0} *$ Resolution & 1,014 \\
\cline { 2 - 3 } & Leaf size $=\mathbf{5} *$ Resolution & 3,036 \\
\cline { 2 - 3 } & Leaf size $=$ Resolution & 17,311 \\
\hline
\end{tabular}

${ }^{*}$ Resolution $=0.01 \mathrm{~m}$ in the case 1 .
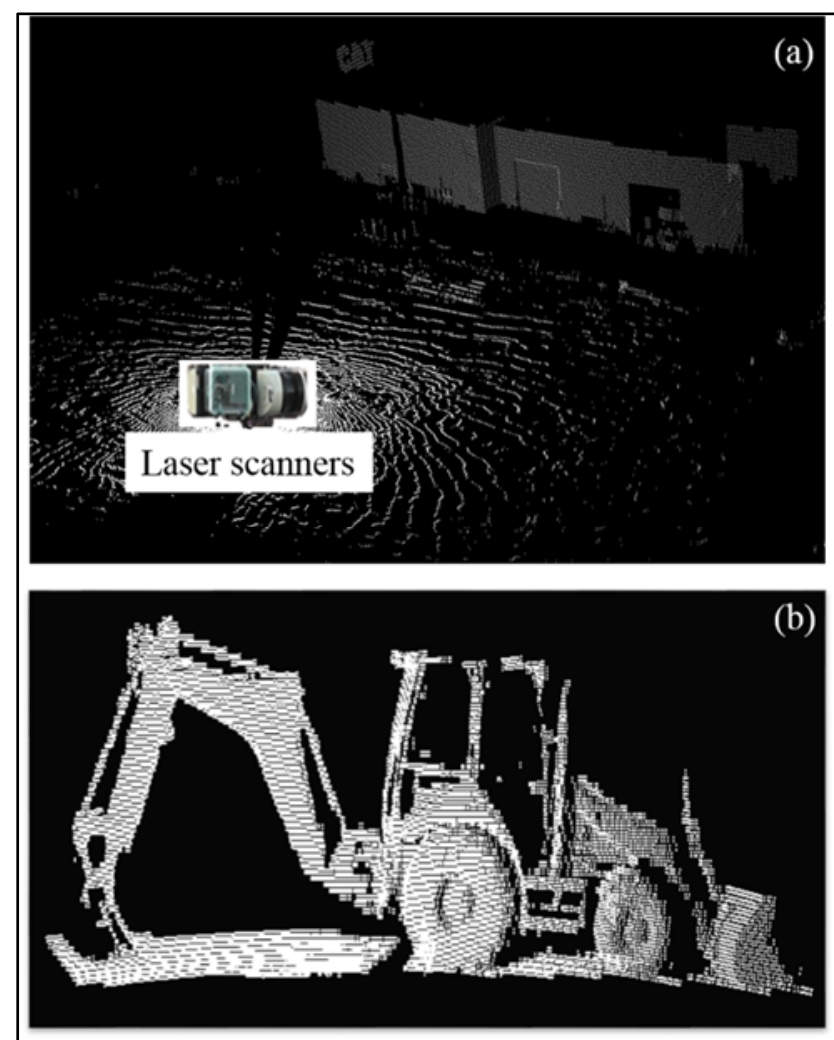

Fig. 4. Collected point cloud of (a) the static site environment and (b) the target area 


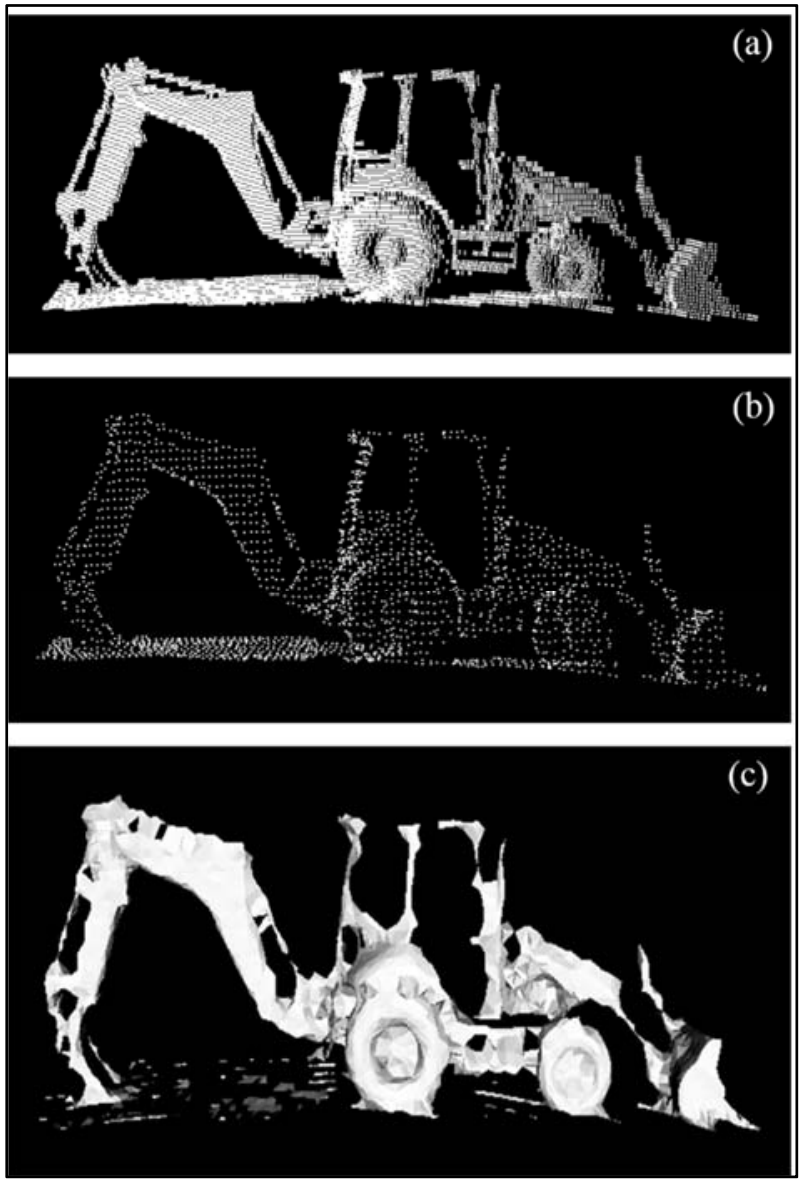

Fig. 5. The point cloud of (a) the filtered data, (b) the downsized data, and (c) the created surface model

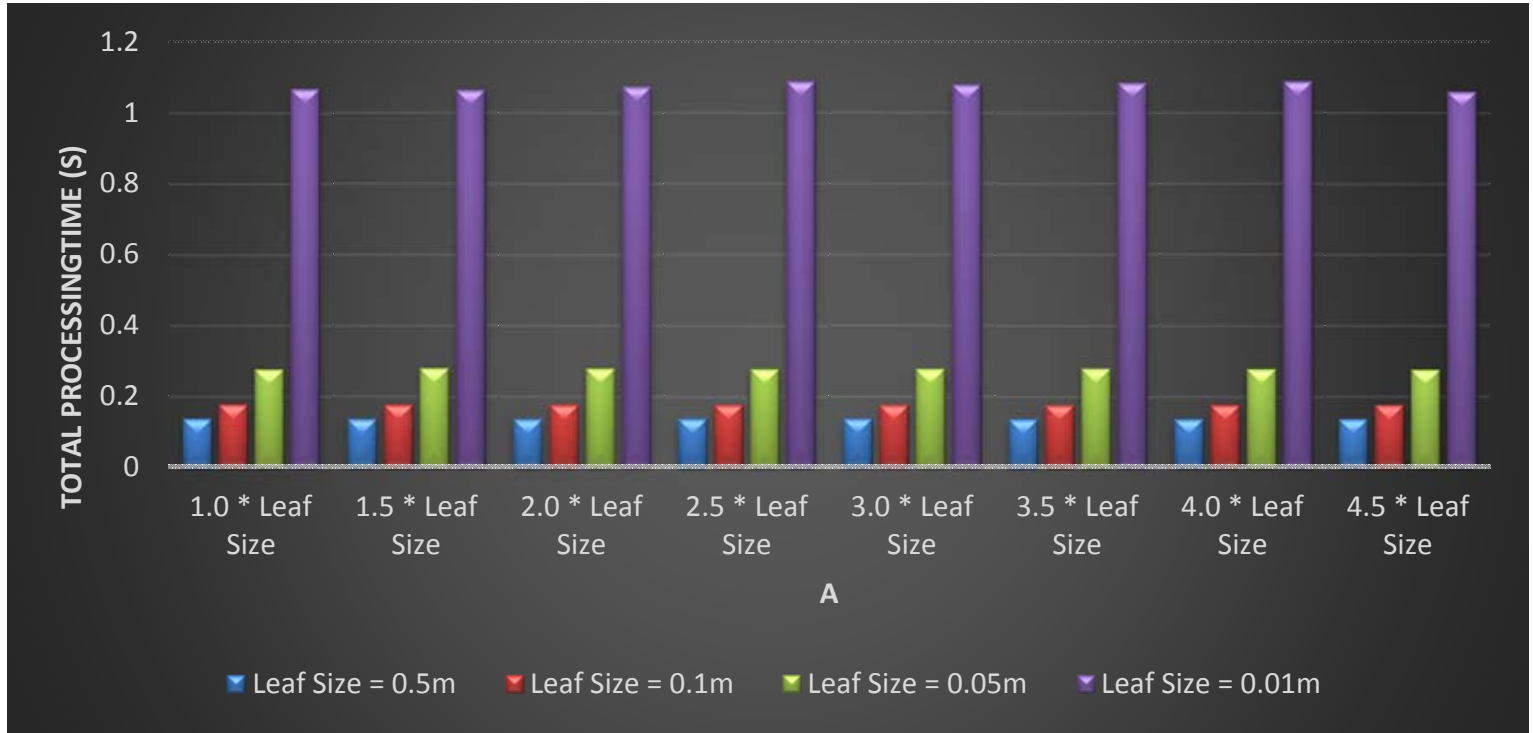

Fig. 6. Total processing time with different $\alpha$ value 
Table 2. Processing time of each step of the proposed methodology

\begin{tabular}{|c|c|c|c|c|c|}
\hline \multirow{2}{*}{\multicolumn{2}{|c|}{ Time (s) }} & \multicolumn{4}{|c|}{ Data Downsizing Leaf Size (m) } \\
\hline & & 0.5 & 0.1 & 0.05 & 0.01 \\
\hline \multicolumn{2}{|c|}{ Data Filtering } & 0.133 & 0.133 & 0.133 & 0.133 \\
\hline \multicolumn{2}{|c|}{ Data Downsizing } & 0.003 & 0.003 & 0.004 & 0.006 \\
\hline \multirow{8}{*}{ 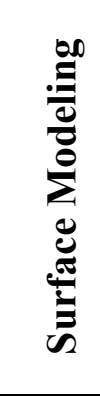 } & $\alpha=1.0 *$ Leaf Size & 0.002 & 0.042 & 0.140 & 0.929 \\
\hline & $\alpha=1.5 *$ Leaf Size & 0.001 & 0.041 & 0.143 & 0.925 \\
\hline & $\alpha=2.0 *$ Leaf Size & 0.001 & 0.041 & 0.142 & 0.935 \\
\hline & $\alpha=2.5 *$ Leaf Size & 0.002 & 0.041 & 0.141 & 0.950 \\
\hline & $\alpha=3.0 *$ Leaf Size & 0.002 & 0.040 & 0.141 & 0.940 \\
\hline & $\alpha=3.5 *$ Leaf Size & 0.001 & 0.040 & 0.143 & 0.946 \\
\hline & $\alpha=4.0 *$ Leaf Size & 0.001 & 0.040 & 0.141 & 0.950 \\
\hline & $\alpha=4.5 *$ Leaf Size & 0.001 & 0.040 & 0.139 & 0.920 \\
\hline
\end{tabular}

Table 3. Test on raw data without filtering and downsizing

\begin{tabular}{|c|c|c|c|c|c|}
\hline $\boldsymbol{\alpha}$ & $\begin{array}{c}\text { Surface } \\
\text { modeling time (s) }\end{array}$ & $\alpha$ & $\begin{array}{c}\text { Surface modeling } \\
\text { time (s) }\end{array}$ & $\boldsymbol{\alpha}$ & $\begin{array}{c}\text { Surface modeling } \\
\text { time (s) }\end{array}$ \\
\hline 0.01 & 1.003 & 0.08 & 0.948 & 0.6 & 1.853 \\
\hline 0.02 & 0.961 & 0.09 & 0.947 & 0.7 & 0.924 \\
\hline 0.03 & 0.965 & 0.1 & 0.948 & 0.8 & 1.854 \\
\hline 0.04 & 0.963 & 0.2 & 1.888 & 0.9 & 2.782 \\
\hline 0.05 & 0.967 & 0.3 & 2.822 & 1.0 & 0.925 \\
\hline 0.06 & 0.962 & 0.4 & 3.752 & & \\
\hline 0.07 & 0.954 & 0.5 & 0.924 & & \\
\hline
\end{tabular}

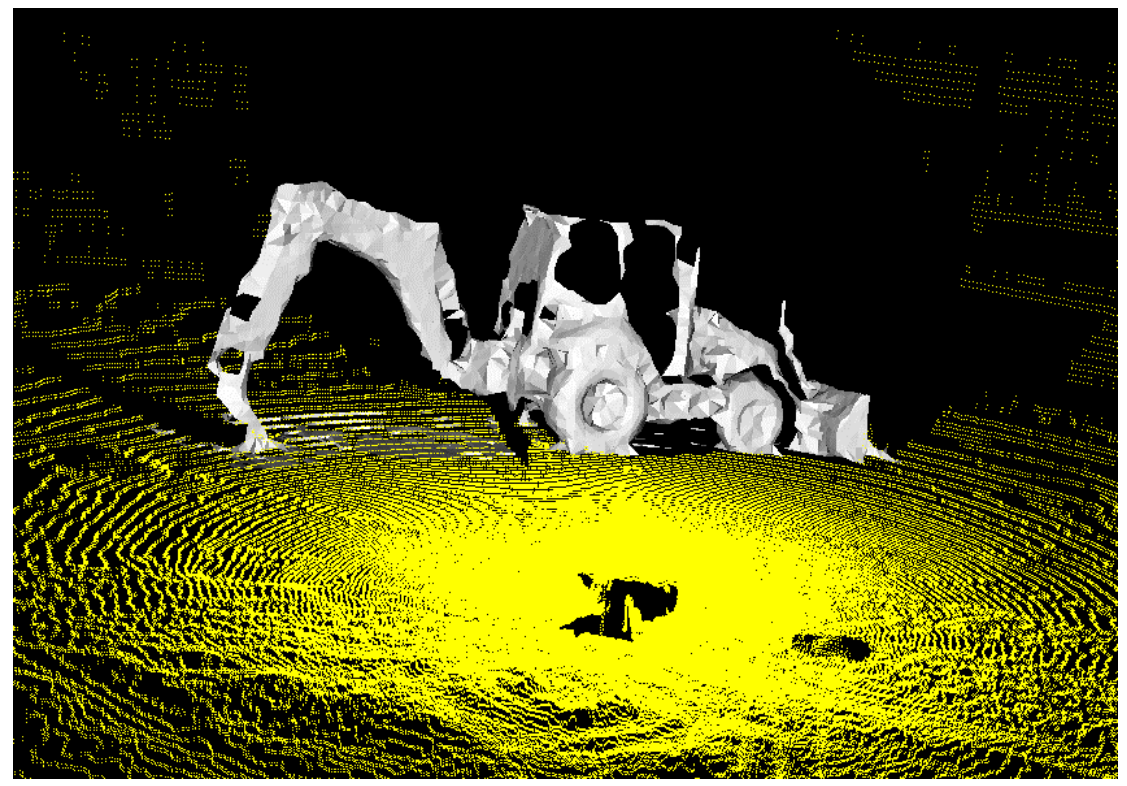

Fig. 7. The created surface model was integrated with the static site environment 


\section{Conclusion}

In this study, a specially designed data capturing system was utilized to collect point cloud data from multiple laser scanners; while multiple video camera arrays were used to rapidly recognize and track the selected dynamic construction equipment objects including a backhoe loader and a crane. The validation of the proposed method was implemented at a real world construction jobsite. The concave hull of the crawler crane was generated in less than 0.5 seconds, and then the data were smoothly transferred to the operator in a cabin. The test results indicate that the proposed rapid workspace modeling approach can improve the heavy equipment operations by distinguishing surface-modeled dynamic target objects from the point cloud of existing static environment in $3 \mathrm{D}$ views in near real time.

While the surface modeling time is sufficiently fast enough for real-time operation, the data collection time should be carefully configured based on the types of equipment (e.g., size and moving speed), distance, ambient lighting, and reflectivity.

For future work, the research will continue to improve the resolution of laser scanner data while reducing data collection time. With an increase in scanning speed, the scanned resolution is lowered accordingly. To resolve this issue, a smart scanning approach with differentiated scan speeds will be further developed, to allow faster rotations for the areas to be skipped, and slow the scan speed for the target areas. Improving surface modeling speed using a higher performance computer and surface model quality will be another future research focus.

\section{Acknowledgement}

This material is based on work supported by the National Science Foundation (Award \#: CMMI1055788). Any opinions, findings, and conclusions or recommendations expressed in this material are those of the authors and do not necessarily reflect the views of the NSF.

\section{References}

[1] H. Son, C. Kim, K. Choi, Rapid 3D object detection and modeling using range data from $3 \mathrm{D}$ range imaging camera for heavy equipment operation, Automation in Construction 19(7) (2010) 898-906.

[2] J. Teizer, B. S. Allread, C. E. Fullerton, J. Hinze, Autonomous pro-active real-time construction worker and equipment operator proximity safety alert system, Automation in Construction 19(5)
(2010) 630-640.

[3] J. Teizer, C. H. Caldas, C. T. Haas, Real-Time Three-Dimensional Occupancy Grid Modeling for the Detection and Tracking of Construction Resources, ASCE Journal of Construction Engineering and Management 133(11) (2007) 880888 .

[4] H. Son, C. Kim, 3D structural component recognition and modeling method using color and $3 \mathrm{D}$ data for construction progress monitoring, Automation in Construction 19(7) (2010) 844-854.

[5] Y. Cho, C. Haas, K. Liapi, S. Sreenivasan, A framework for rapid local area modeling for construction automation, Automation in Construction 11(6) (2002) 629-641.

[6] Y. Cho, C. Haas, S. Sreenivasan, K. Liapi, Error Analysis and Correction for Large Scale Manipulators in Construction, ASCE Journal of Construction Engineering and Management 130 (1) (2004) 50-58.

[7] Y. Cho, C. Wang, P. Tang, C. Haas, Targetfocused local workspace modeling for construction automation applications, ASCE Journal of Computing in Civil Engineering 26(5) (2012) 661670.

[8] Y. Cho, C. Haas, Rapid Geometric Modeling for Unstructured Construction Workspaces, Journal of Computer-Aided Civil and Infrastructure Engineering 18 (2003) 242-253.

[9] J. Gong, C. H. Caldas, Data processing for realtime construction site spatial modeling, Automation in Construction 17(5) (2008) 526-535.

[10] P. Tang, D. Huber, B. Akinci, R. Lipman, Automatic reconstruction of as-build building information models from laser-scanned point clouds: A review of related techniques, Automation in Construction 19 (2010) 829-843.

[11] D. Huber, B. Akinci, P. Tang, A. Adan, Using laser scanner for modeling and analysis in architecture, engineering and construction, Proceedings of the Conference on Information Sciences and Systems (CISS) (2010) Princeton, NJ.

[12] C. Kim, J. Lee, M. Cho, C. Kim, Fully automated registration of $3 \mathrm{D}$ CAD model with point cloud from construction site, 28th International Symposium on Automation and Robotics in Construction, Seoul, Korea, (2011) 917-922.

[13] G. Lee, H. Kim, C. Lee, S. Ham, S. Yun, H. Cho, B. Kim, G. Kim, K. Kim, A laser-technologybased lifting-path tracking system for a robotic 
tower crane, Automation in Construction 18(7) (2009) 865-874.

[14] T. M. Ruff, Recommendations for evaluating \& implementing proximity warning systems on surface mining equipment, Research Report to the National Institute for Occupational Safety and Health, Centers for Disease Control, (2007) < http://www.cdc.gov/niosh/mining/works/covershee t202.html >.

[15] I. Brilakis, M. Park, G. Jog, Automated vision tracking of project related entities, Advanced Engineering Informatics 25 (2011) 713-724.

[16] M. Park, C. Koch, I. Brilakis, Three-dimensional tracking of construction resources using an on-site camera system, J. Comput. Civ. Eng. 26(4) (2012) 541-549.

[17] Y. Cho, M. Gai. Projection-Recognition-Projection (PRP) Method for Rapid Object Recognition and Registration from a 3D Point Cloud, ASCE Journal of Computing in Civil Engineering (2014) doi: 10.1061/(ASCE) CP.1943-5487.0000332 (in press).

[18] C. Wang, Y. Cho, M. Gai, As-is 3D Thermal Modeling for Existing Building Envelopes Using a Hybrid LIDAR System, ASCE Journal of Computing in Civil Engineering 27(6) (2013) 645656.

[19] Y. Arayici, An approach for real world data modeling with the $3 \mathrm{D}$ terrestrial laser scanner for built environment, Automation in Construction 16 (6) (2007) 816-829.

[20] F. Bosche, C. T. Haas, Automated retrieval of 3D CAD model objects in construction range images, Automation in Construction 17(4) (2008) 499-512.

[21] H. Bay, T. Tuytelaars, L. V. Gool, SURF: Speeded up robust features, Computer Vision-ECCV (2008) 404-417.

[22] P. Zhou, Computational Geometry: Analysis and Design on the Algorithms, second edition, Tsinghua University press, Beijing, 2005.

[23] C. Kim, C. Haas, K. Liapi, C. Caldas, HumanAssisted Obstacle Avoidance System Using 3D Workspace Modeling for Construction Equipment Operation, ASCE J. Comp. in Civ. Engrg. 20(3) (2006) 177-186. 